\title{
Video Article \\ Assessment of Cerebral Lateralization in Children using Functional Transcranial Doppler Ultrasound (fTCD)
}

\author{
Dorothy V. M. Bishop ${ }^{1}$, Nicholas A. Badcock ${ }^{1}$, Georgina Holt ${ }^{1}$ \\ ${ }^{1}$ Department of Experimental Psychology, University of Oxford \\ Correspondence to: Dorothy V. M. Bishop at dorothy.bishop@psy.ox.ac.uk
}

URL: https://www.jove.com/video/2161

DOI: doi:10.3791/2161

Keywords: Neuroscience, Issue 43, functional transcranial Doppler ultrasound, cerebral lateralization, language, child

Date Published: 9/27/2010

Citation: Bishop, D.V., Badcock, N.A., Holt, G. Assessment of Cerebral Lateralization in Children using Functional Transcranial Doppler Ultrasound (fTCD). J. Vis. Exp. (43), e2161, doi:10.3791/2161 (2010).

\section{Abstract}

There are many unanswered questions about cerebral lateralization. In particular, it remains unclear which aspects of language and nonverbal ability are lateralized, whether there are any disadvantages associated with atypical patterns of cerebral lateralization, and whether cerebral lateralization develops with age. In the past, researchers interested in these questions tended to use handedness as a proxy measure for cerebral lateralization, but this is unsatisfactory because handedness is only a weak and indirect indicator of laterality of cognitive functions ${ }^{1}$. Other methods, such as fMRI, are expensive for large-scale studies, and not always feasible with children ${ }^{2}$.

Here we will describe the use of functional transcranial Doppler ultrasound (FTCD) as a cost-effective, non-invasive and reliable method for assessing cerebral lateralization. The procedure involves measuring blood flow in the middle cerebral artery via an ultrasound probe placed just in front of the ear. Our work builds on work by Rune Aaslid, who co-introduced TCD in 1982, and Stefan Knecht, Michael Deppe and their colleagues at the University of Münster, who pioneered the use of simultaneous measurements of left- and right middle cerebral artery blood flow, and devised a method of correcting for heart beat activity. This made it possible to see a clear increase in left-sided blood flow during language generation, with lateralization agreeing well with that obtained using other methods ${ }^{3}$.

The middle cerebral artery has a very wide vascular territory (see Figure 1) and the method does not provide useful information about localization within a hemisphere. Our experience suggests it is particularly sensitive to tasks that involve explicit or implicit speech production. The 'gold standard' task is a word generation task (e.g. think of as many words as you can that begin with the letter ' $\mathrm{B}$ ') ${ }^{4}$, but this is not suitable for young children and others with limited literacy skills. Compared with other brain imaging methods, fTCD is relatively unaffected by movement artefacts from speaking, and so we are able to get a reliable result from tasks that involve describing pictures aloud ${ }^{5,6}$. Accordingly, we have developed a child-friendly task that involves looking at video-clips that tell a story, and then describing what was seen.

\section{Video Link}

The video component of this article can be found at https://www.jove.com/video/2161/

\section{Protocol}

\section{Using fTCD to Assess Language Lateralization in Children}

1. Where possible, we familiarise the child with pictures showing characters from the video stories in a separate session before introducing them to Doppler system.

2. It is important that the procedure includes sufficient trials to get a reliable measure: ideally 20 or more, though we have obtained useful data with as few as 10 to 15 trials. Each trial involves a period of relaxation, to be used as a baseline, followed by language activation. Blood flow velocity during the period of activation is compared against the baseline. We have found that it is possible to use the period while the child passively watches a 12 second video clip as a baseline. This helps avoid boredom. The baseline is followed by a 10 second response period in which the child is asked to describe what happened. This is followed by a 16 second rest period.

3. The timing of the procedure is important in order to detect changes in blood flow velocity due to metabolic demands, which may take up to 7 seconds. We use computerised tasks, written in Presentation or Matlab, which control the timing of each trial. Critically, these programs also send pulses to the hardware which records a marker in the Doppler data file to indicate onset of the activation period. These are used in the data analysis to determine periods of baseline and activation blood flow.

4. We use a Multi-dop system which enables ultrasound recording from left and right sides simultaneously. It is best to have the Multi-dop system set up and ready to go before the participant arrives. Make sure the probes are connected to the Multi-dop system before switching on. Check that the stimulus computer is connected, via parallel port, to the Multi-dop computer and pulses from one are seen on the other. Ensure the highpass filter is set to $300 \mathrm{~Hz}$ for both sides and check the depth of insonation (45-55 mm) and the power of the signal. The system has limits set on the different parameters to ensure only safe levels can be used.

5. A participant code should be recorded in the Multi-dop data files in order for condition, group, or demographic data matching. 
6. The system should then be paused while preparing the probes. Each ultra-sound probe is covered with gel which provides a contact between the probe and the skin. Use a generous amount of gel to maintain the best contact possible.

7. Now it is time to start the session. Check whether the child needs to go to the toilet before starting! We demonstrate the headset using toy animals, and make sure that the child understands about what is going to happen and what they will be asked to do. It is particularly important that they understand the need to keep quiet after the activation period, so that the signal can return to baseline. Children should be seated comfortably as a full task may last between 20 and 30 minutes and a lot of gross body movements can interfere with the Doppler signal.

8. The hardest part of the procedure is the positioning of the gel-covered probes. New users should be aware that it takes practice to become expert at this part of the process. There is a useful computer simulation by Rune Aaslid that can help with training

(http://www.transcranial.com/), but the best way to learn is by practising on friends and colleagues. Once you have assessed about 10 people, you will start to find it much easier. One good thing about working with children is that it is usually easier to find a signal from a child than an adult, because they have thinner skulls.

9. Each probe is placed at the temporal window of the skull on each side of the head. This is the thinnest section of bone in the head and provides the best area through which a signal can be obtained from the middle cerebral arteries. These windows can be found just forward of each ear. When working with children, it can help reduce the set-up time if you have two experimenters, one adjusting each probe. Most children are happy to watch a DVD during this part of the procedure, but it is important to monitor the child's behavior and ensure they are comfortable and relaxed. Terminate the session if the child requests this or if there is any sign of distress.

10. The position of the probes is then adjusted in order to insonate the middle cerebral artery. It may take a few minutes before a satisfactory signal is found. This signal has a characteristic sound as well as visual pattern which should be monitored from the Multi-dop computer.

11. The characteristic sound is a low whooshing noise of the rate of the participant's heart beat. The characteristic visual pattern is a sharp rise followed by an exponential decay fall off which also repeats at the rate of the heart beat. You may pick up activity from other blood vessels, depending on the depth of insonation and direction of the probe. A depth of approximately 45 to $50 \mathrm{~mm}$ usually works well in children. Once a strong signal has been found, reduce the power as much as possible while maintaining a good signal. The gain can also be increased to amplify a weak signal.

12. Once the signals for each middle cerebral artery are satisfactory, the auditory output of the Multi-dop signal should be turned down, and the procedure can begin. The experimenter explains to the child that they are going to watch a video clip quietly. When they see the question mark come up on the computer they need to tell the experimenter as much as they can about what happened in the video. When they see the picture of the boy going 'shhh' they have to stop talking immediately and sit quietly to wait for the next video to start (see Figure 2 and .avi examples).

13. Recording devices are then started, i.e., a voice recorder for auditory responses; along with the Multi-dop system recording; and the computer controlling stimulus presentation.

14. The experimenter's job is then to monitor the child and Doppler signal to a) make sure the child is comfortable and attending to the task and b) ensure that a suitable signal is maintained. The Doppler signal is far less sensitive to movement than an fMRI signal, but it can still be disrupted by gross body movements, or if the probe moves. If the signal becomes significantly disrupted, the display computer should be paused and the probes reset appropriately. At times it may be necessary to apply more gel in order to gain re-establish a satisfactory signal. We find that testing of children works best if one experimenter is responsible for keeping the child on task and recording what they say, and the other monitors the Doppler signal.

15. When the experimental procedure is finished, the Multi-dop recording can be stopped and the probes removed. It is useful to have some tissues available to remove any excess gel.

16. The raw data files are processed in order to compare the left and right Doppler signals. We have developed our own Matlab software based on the methods developed by Deppe ${ }^{7}$. This processing involves down-sampling the data and correcting for fluctuations due to heart beat. Single trials are excluded from the analysis if the left or right activation is outside a specified range, commonly 70 to $130 \%$ of baseline blood flow velocity. The activation in these trials is likely to be affected by problematic probe contact. Figure 3 shows activation relative to probe signals for a good signal, whereas Figure 4, shows a case where the signal is periodically lost.

17. Relative to baseline blood flow velocity, activation in the right channel is subtracted from the left in order to calculate the difference activation (see Figure 5). The lateralization index is calculated as the average of the difference activation for one second either side of the maximum difference within a pre-defined period of interest. This period of interest is 7 to 17 seconds following the start of the 'talk' command, indicated by the marker sent from the display computer to the Multi-dop data file. As the difference activation is the left minus right channels, positive lateralization indices reflect left lateralization and negative reflect right.

\section{Representative Results}

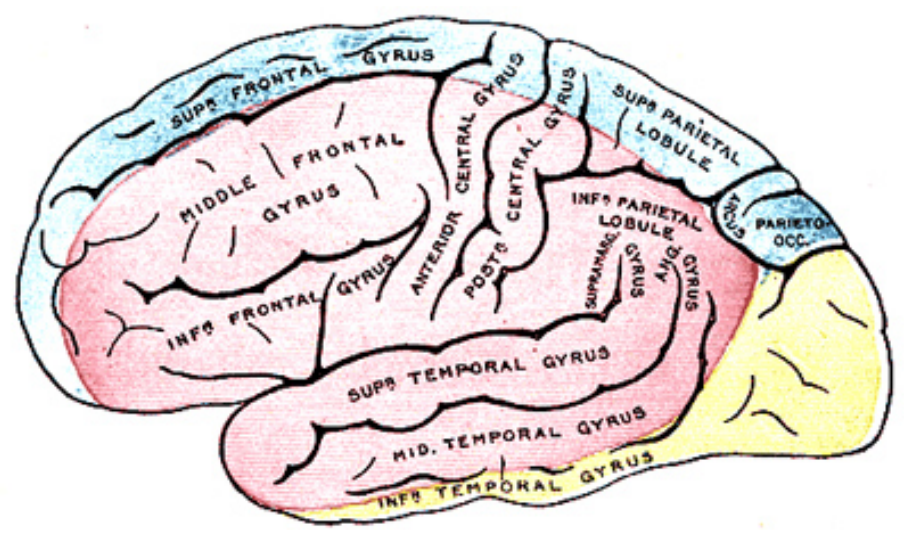


Figure 1: Outer surface of cerebral hemisphere, showing areas supplied by cerebral arteries. Pink is region supplied by middle cerebral artery. (from: http://en.wikipedia.org/wiki/File:Gray517.png)

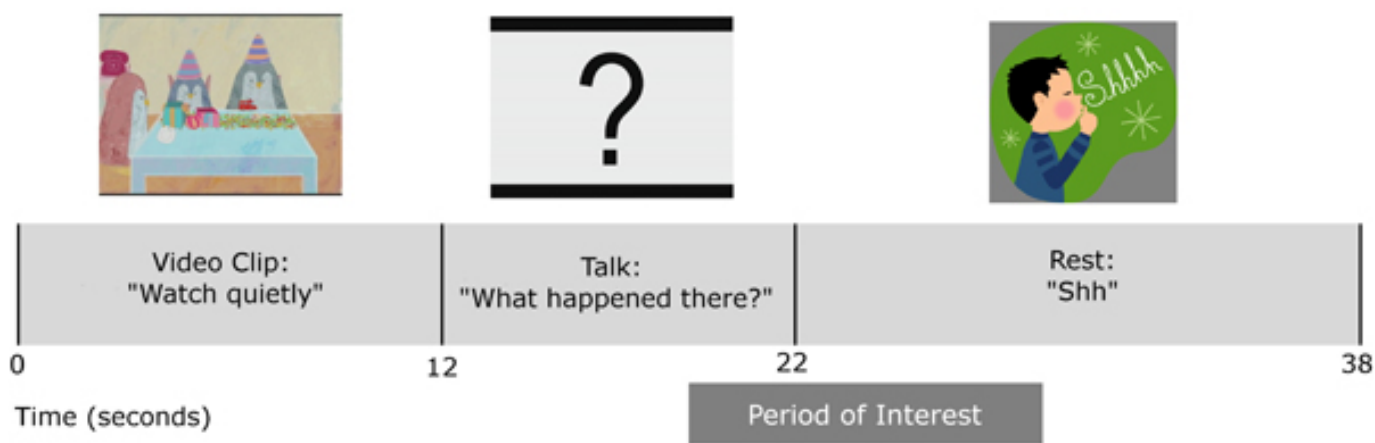

Figure 2: Schematic outline of experimental trial with 12 second baseline video, 10 second response interval, and 16 second rest phase. Period of interest for laterality calculation is also depicted.

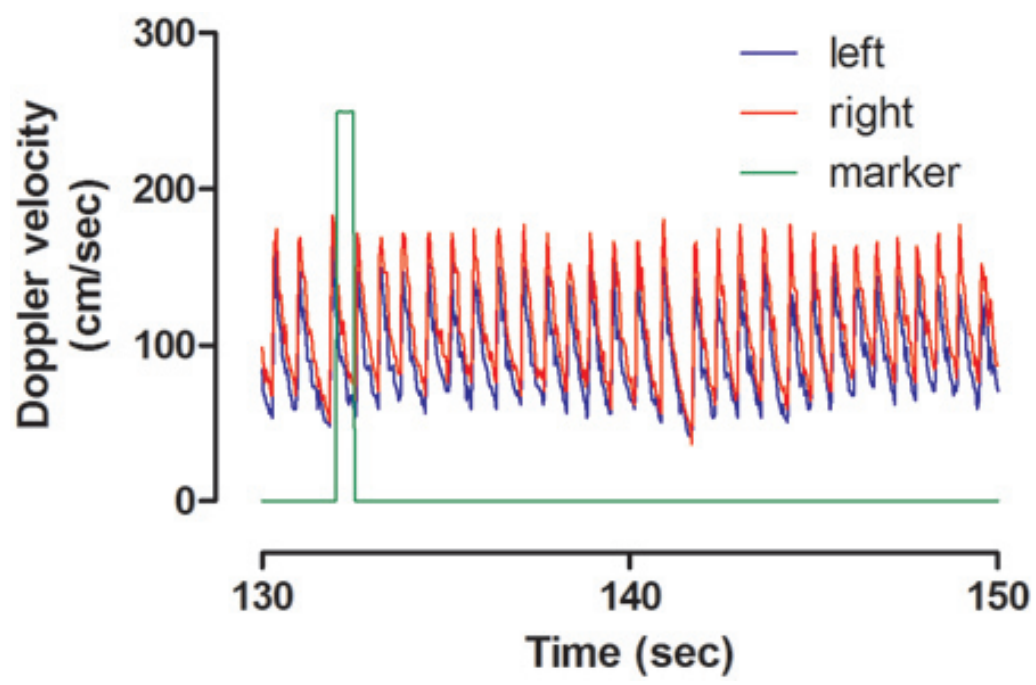

Figure 3. Clean data recording of raw Doppler velocity $(\mathrm{cm} / \mathrm{sec})$ for the Left (blue) and Right(red) Doppler channels from 130 to 150 seconds during a single experimental session. Each visible pulse corresponds to one heart beat. Event marker indicating stimulus onset displayed in green.

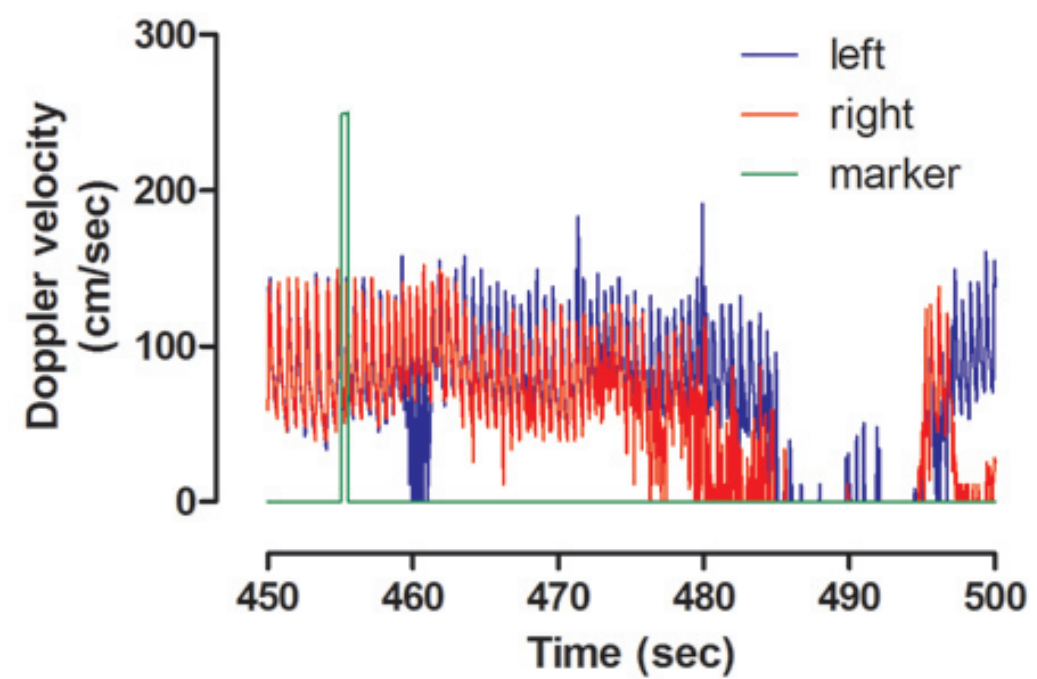

Figure 4. Disrupted data recording of raw Doppler velocity (cm/sec) for the Left (blue) and Right (red) Doppler channels from 400 to 450 seconds during a single experimental session. Event marker indicating stimulus onset displayed in green. Significant evidence of dropout is visible for both channels. 

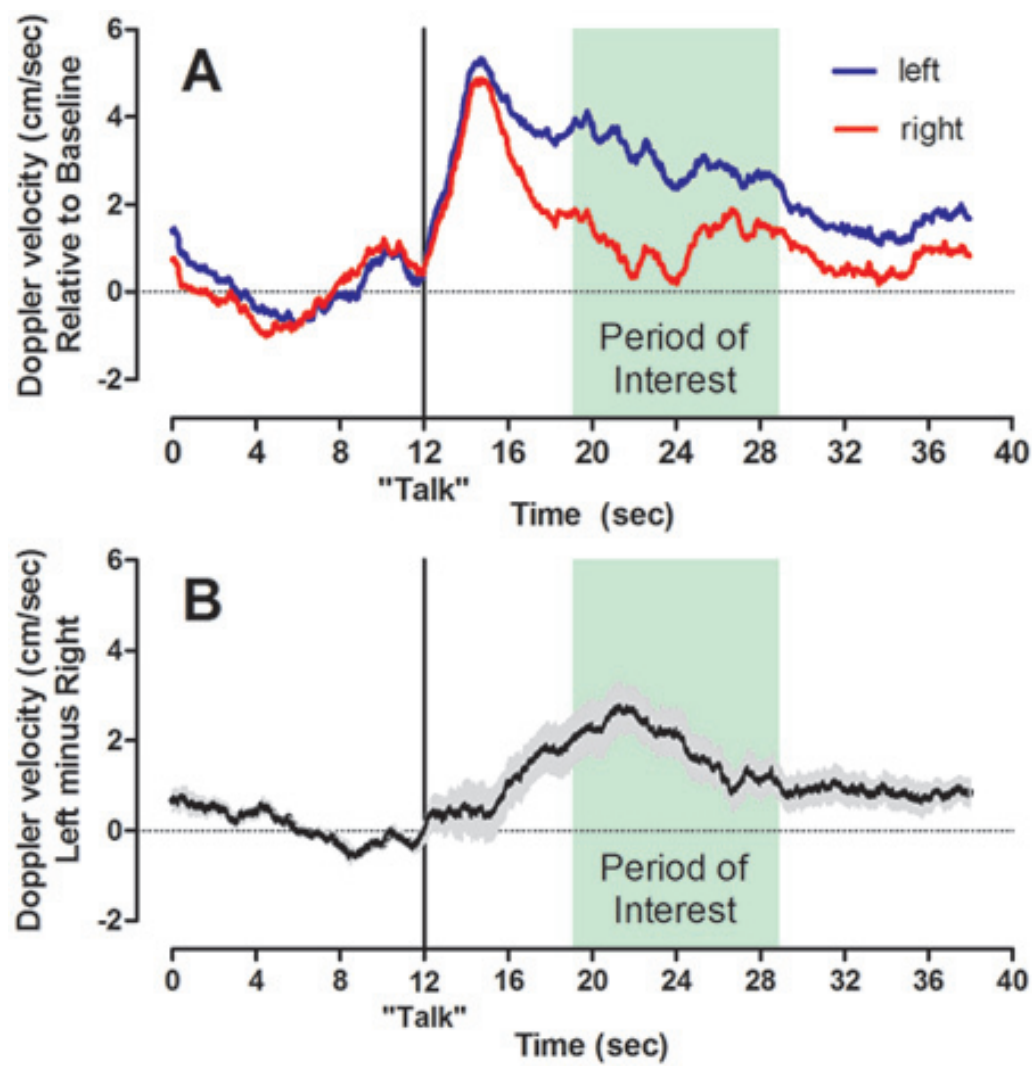

Figure 5. Left (blue) and right (red) channel (Panel A) and left minus right difference (Panel B) Doppler Velocity (cm/sec) averaged across all acceptable trials for a group of participants. Participants were cued to "Talk" at 12 seconds and the period of interest for the laterality calculation is also depicted in green. The grey error bars surrounding the difference velocity in Panel B represent the standard error of the mean.

Avi examples: the video sequences to tell the "Freezefoot" story can be downloaded from: http://psyweb.psy.ox.ac.uk/oscci/Miscellaneous.htm

\section{Discussion}

As well as studying normal development, we have been using fTCD to study language lateralization in adults and children with developmental disorders of language and literacy ${ }^{8,9}$. We are also interested in developing methods of using fTCD for assessment of lateralization of visuospatial abilities ${ }^{10,11}$.

\section{Disclosures}

No conflicts of interest declared.

\section{Acknowledgements}

We thank Hubertus Lohmann for sharing his expertise in fTCD techniques. This work was funded by Wellcome Trust programme grant no. 082498/Z/07/2.

\section{References}

1. Bishop, D. V. M. Handedness and developmental disorder. Blackwell Scientific and Philadelphia: J.B. Lippincott (1990).

2. Pelletier, I., Sauerwein, H., Lepore, F., Saint-Amour, D. \& Lassonde, M. Non-invasive alternatives to the Wada test in the presurgical evaluation of language and memory functions in epilepsy patients. Epileptic Disorders 9, 111-126 (2007).

3. Deppe, M., Ringelstein, E. B. \& Knecht, S. The investigation of functional brain lateralization by transcranial Doppler sonography. Neurolmage 21, 1124-1146 (2004).

4. Knecht, S. et al. Noninvasive determination of language lateralization by functional transcranial Doppler sonography : A comparison with the Wada test. Stroke 29, 82-86 (1998).

5. Lohmann, H., Drager, B., Muller-Ehrenberg, S., Deppe, M. \& Knecht, S. Language lateralization in young children assessed by functional transcranial Doppler sonography. Neuroimage 24, 780-790 (2005). 
6. Bishop, D.V.M., Watt, H. \& Papadatou-Pastou, M. An efficient and reliable method for measuring cerebral lateralization during speech with functional transcranial Doppler ultrasound. Neuropsychologia 47, 587-590 (2009).

7. Deppe, M., Knecht, S., Henningsen, H. \& Ringelstein, E.B. AVERAGE: a Windows program for automated analysis of event related cerebral blood flow. Journal of Neuroscience Methods 75, 147-154 (1997).

8. Whitehouse, A.J.O. \& Bishop, D.V.M. Cerebral dominance for language function in adults with specific language impairment or autism. Brain $131,3193-3200(2008)$.

9. Illingworth, S. \& Bishop, D.V. Atypical cerebral lateralisation in adults with compensated developmental dyslexia demonstrated using functional transcranial Doppler ultrasound. Brain and Language 111, 61-65 (2009)

10. Whitehouse, A. J. O. \& Bishop, D. V. Hemispheric division of function is the result of independent probabilistic biases. Neuropsychologia 47, 1938-1943 (2009).

11. Whitehouse, A.J.O., Badcock, N., Groen, M.A. \& Bishop, D.V.M. Reliability of a novel paradigm for determining hemispheric lateralization of visuospatial function. Journal of the International Neuropsychological Society 15, 1028-1032 (2009). 